\title{
Soft Inextensibility Constraints for Template-Free Non-rigid Reconstruction ${ }^{\star}$
}

\author{
Sara Vicente and Lourdes Agapito \\ School of Electronic Engineering and Computer Science, \\ Queen Mary University of London, Mile End Road, London E1 4NS, UK \\ \{sara.vicente, lourdes.agapito\}@eecs.qmul.ac.uk
}

\begin{abstract}
In this paper, we exploit an inextensibility prior as a way to better constrain the highly ambiguous problem of non-rigid reconstruction from monocular views. While this widely applicable prior has been used before combined with the strong assumption of a known 3Dtemplate, our work achieves template-free reconstruction using only inextensibility constraints. We show how to formulate an energy function that includes soft inextensibility constraints and rely on existing discrete optimisation methods to minimise it. Our method has all of the following advantages: (i) it can be applied to two tasks that have been so far considered independently - template based reconstruction and non-rigid structure from motion - producing comparable or better results than the state-of-the art methods; (ii) it can perform template-free reconstruction from as few as two images; and (iii) it does not require post-processing stitching or surface smoothing.
\end{abstract}

Keywords: Non-rigid reconstruction, inextensiblility priors, MRF optimization.

\section{Introduction}

The reconstruction of non-rigid 3D objects from monocular sequences is an inherently ambiguous problem that has received a significant amount of attention in recent years. Most methods for reconstruction of deformable objects fall into two categories: template based methods [1-3], where the 3D shape of the object is known for a reference frame; and methods that rely on points being tracked along a video sequence, such as the pioneer non-rigid structure from motion (NRSfM) methods based on the statistical prior that the shape of a non-rigid object can be expressed in a compact way as a linear combination of an unknown low-rank shape basis $[4,5]$.

In both cases, additional priors or assumptions have to be imposed to better constrain a highly ambiguous problem. These priors vary in their applicability range. Broadly applicable priors include temporal smoothness $[5,6]$ and surface

* This work was funded by the European Research Council under the ERC Starting Grant agreement 204871-HUMANIS. 
(a) Input images with correspondences

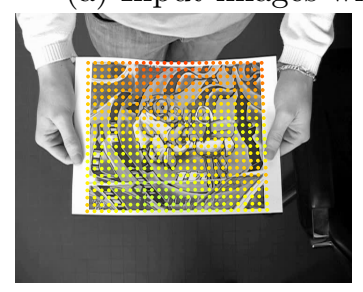

(b) 3D Reconstruction

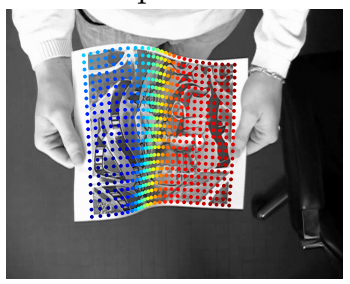

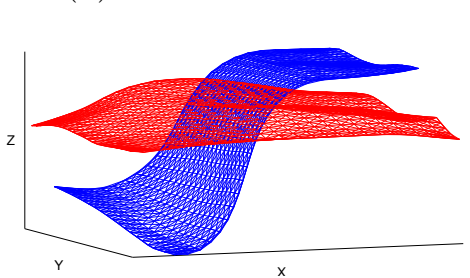

Fig. 1. 3D Reconstruction obtained using our template-free method based on soft inextensibility constraints. Given (a) two input images and point correspondences (colour coded according to their estimated depth), (b) shows the estimated $3 \mathrm{D}$ reconstructions estimated using our approach. Best viewed in colour.

smoothness $[2,3]$. Temporal smoothness is particularly meaningful for reconstruction from video sequences, where there are only small deformations from one frame to the next, but it relies on having coherent tracks for the full sequence. Surface smoothness is also a commonly used prior, but in general it is too weak to be used alone.

In this paper we consider a more restrictive, but still widely applicable prior: an inextensibility constraint. A surface is inextensible if it does not stretch or shrink, i.e. if the geodesic distances between points are preserved under deformations. This is satisfied in a variety of situations, for example, if the images depict an inextensible material (e.g. paper and non-stretchable fabric) or if the object is articulated.

Inextensibility constraints have been extensively used for template based reconstruction [1-3], but less explored when no template is available. The assumption that a $3 \mathrm{D}$ template shape is available is quite restrictive and in practice template based methods have mostly been applied to surfaces which are isometric with a plane, i.e. developable surfaces. This may be related to the difficulty in obtaining an accurate template for other types of surfaces.

Besides not requiring a template shape our method uses a soft version of the inextensibility constraint. Instead of enforcing the constraint exactly, we penalise deviations from it. There are several scenarios in which a soft constraint is more adequate, for instance when there is a small level of stretching but inextensibility is still broadly observed. Moreover, a soft constraint will result in improved robustness in the presence of noise.

Inextensibility constraints are usually imposed in a small neighbourhood, such that the distances of nearby points are preserved in the $3 \mathrm{D}$ reconstruction. This can be interpreted as enforcing local rigidity. Local rigidity constraints have been previously considered in $[7,8]$. Both methods form part of a recent trend of piecewise reconstruction methods in NRS $f$ M. Instead of relying on a single model for the full surface, these approaches model small patches of the surface independently. The main advantage of piecewise modelling is that complex surfaces can still be recovered, while keeping the complexity of each local model low. On 
the other hand, since each patch is reconstructed independently, these methods require a post-processing stitching step. Compared to prior work, ours is the first to include all of the following advantages:

It does not require a template shape, in contrast to most previous methods that impose inextensibility constraints. Instead we rely on having point correspondences between images.

It does not require a temporal sequence, instead we require as few as two images and do not use information about their temporal order.

It imposes inextensibility as a soft constraint. A soft constraint guarantees improved robustness.

It does not require post-processing stitching, in contrast to existing piecewise reconstruction methods where patches are reconstructed independently and then require a further 3D alignment step.

In this paper we propose a pointwise reconstruction method that recovers the depth of each interest point in each image. We formulate the task of 3D reconstruction under inextensibility constraints as an energy minimisation problem, where the energy is a function of the depths. This energy is minimised using discrete optimisation techniques. Besides inferring the depth for all points we also recover a weak template of the underlying shape. An example of the application of our method is shown in Fig. 1. Given two images with point correspondences shown in (a), our method recovers the 3D shape shown in (b) by imposing inextensibility constraints without the need for a known template shape.

\section{$1.1 \quad$ Related Work}

The inextensibility prior has been most commonly used in template based methods. These focus on reconstructing a single image, assuming that correspondences between the image and a 3D template shape are given. Template based methods have been mostly applied to the reconstruction of developable surfaces. Since developable surfaces are isometric with a plane, the template shape is usually a planar surface. A closed form solution for this problem was introduced in [1], where the template was in the form of a $2 \mathrm{D}$ mesh and the inextensibility constraint was enforced between mesh nodes. A different version of the inextensibility constraint that allows shrinking to account for the differences between Euclidean and geodesic distances was presented in [9] and shown to be more suitable for reconstructing sharply folding surfaces. In [2] the known distances between interest points were used to obtain upper bounds on the depth of each point. This approach was later extended in [3] to account for noise in the correspondences and in the template.

An interesting exception to this coupling between inextensibility priors and template based methods was presented in [10]. This method assumed the surface was developable and recovered a planar template given correspondences across multiple images. In contrast to these methods our approach is not only template free, but also applicable to non-developable surfaces.

Also related to our approach are the recently introduced piecewise models [7, $8,11,6]$. In general, these methods can cope with sequences with stronger deformations than previous global approaches. They differ in the type of local model 


\begin{tabular}{l|l}
\hline Notation & Description \\
\hline$S$ & Number of input images. \\
\hline$N$ & Number of interest points. We use $i$ and $j$ to index the set of points. \\
\hline$p_{i}^{s}=\left(x_{i}^{s}, y_{i}^{s}\right)$ & 2D point $i$ in image frame $s$. \\
\hline$Q_{i}^{s}$ & 3D coordinates of point $i$ on the shape corresponding to frame $s$. \\
\hline$\mu_{i}^{s}$ & Depth of the 3D point $i$ on the shape corresponding to frame $s$. \\
\hline $\mathcal{N}=\{(i, j)\}$ & $\begin{array}{l}\text { Neighbourhood system: the set of all pairs }(i, j) \text { of neighbouring } \\
\text { points. Points } i \text { and } j \text { are neighbours if their image distance is small } \\
\text { in all frames. }\end{array}$ \\
\hline
\end{tabular}

Table 1. Notation. When referring to the case of a single input image or a generic image, we sometimes drop the index $s$.

considered (piecewise planarity [7], local rigidity [8] and quadratic deformations $[11,6])$ and in the way the surface is divided into patches. For example in [8] each patch is a triangle formed by neighbouring points, while in [7] the surface is divided into regular rectangular patches. A more principled approach was proposed in [6], where the format of the patches is jointly optimised with the model for each patch.

Inextensibility is imposed in the piecewise planar patches used in [7] and the rigid triangles used in [8]. Neither of these approaches require a template. However, they both require a stitching step to recover a coherent final surface. Furthermore, [7] requires a mesh fitting step with manual initialisation to recover a smooth surface, while the method in [8] discards non-rigid triangles, producing only partial reconstructions. Our method does not suffer from any of these disadvantages. It produces a full reconstruction, even for parts which violate the inextensibility constraint, and does not require any post-processing step of stitching or mesh fitting.

Most related to our approach is the "incremental rigidity scheme" proposed in [12] for temporal sequences. The scheme is applicable to both rigid and nonrigid objects and it maintains a $3 \mathrm{D}$ model of the object which is incrementally updated based on every new frame, by maximizing rigidity. Since the initialization of the model consists of a flat shape (at constant depth), the method is not expected to recover a plausible reconstruction for the initial frames. This scheme was later extended in [13] to reconstruct deforming developable surfaces. Our method differs from these approaches in that we aim at recovering the $3 \mathrm{D}$ shape corresponding to all frames and we do not require a temporal sequence.

\subsection{Notation}

We start by defining the notation used throughout the paper and discuss some of the assumptions made. Our notation is summarised in Table 1.

As previously mentioned, a surface is inextensible if the geodesic distances between points are preserved under deformation. Since our method performs pointwise 3D reconstruction (as opposed to recovering a continuous surface) we do not have access to the geodesic distance between points. Instead we rely on 
(a) Orthographic camera

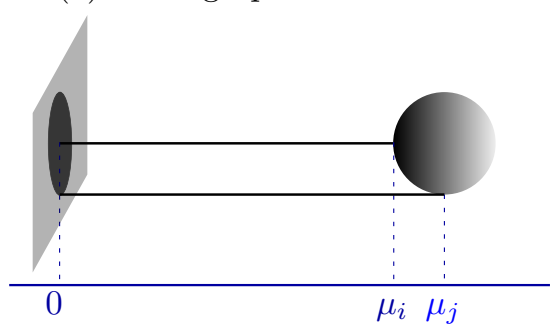

(b) Perspective camera

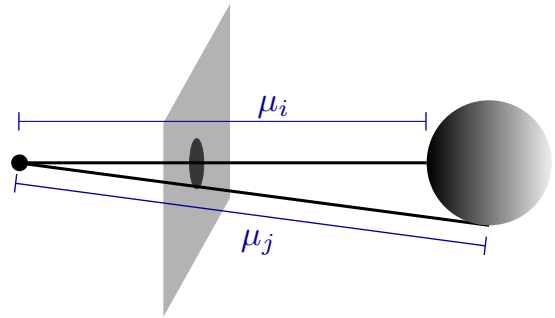

Fig. 2. Illustration of the camera models considered. In both cases, we reduce the problem of $3 \mathrm{D}$ reconstruction of point $i$ to finding its depth $\mu_{i}$.

the Euclidean distance being a reasonable approximation. We consider distances between nearby points, making this approximation more meaningful than previous approaches that considered it for all possible pairs of points $[2,3]$.

For simplicity, we consider we have a single static camera and explore the cases of both orthographic and calibrated perspective cameras. Similarly to [2], we assume that the world frame is aligned with the camera coordinate system and that the noise in the internal calibration is small so that the $3 \mathrm{D}$ reconstruction reduces to finding the depth of the point in each image.

Orthographic camera Under orthographic projection, a 3D point $Q_{i}$ can be written as a function of its depth $\mu_{i}$ as: $Q_{i}\left(\mu_{i}\right)=\left(x_{i}, y_{i}, \mu_{i}\right)$. This is illustrated in Fig. 2. Orthography is a simplifying assumption applicable when the relief of the object is small compared with the distance to the camera.

Perspective camera For a calibrated perspective camera, a 3D point is given by $Q_{i}\left(\mu_{i}\right)=\mu_{i} v_{i}$, where $v_{i}$ is the direction of the sightline defined as $v_{i}=$ $\frac{M^{-1} \bar{p}_{i}}{\left\|M^{-1} \bar{p}_{i}\right\|}$, where $\bar{p}_{i}$ is the point $p_{i}$ in homogeneous coordinates and $M$ is the matrix of intrinsic camera parameters.

The goal of our 3D reconstruction approach is to recover the depths $\mu_{i}^{s}$ and corresponding 3D points $Q_{i}^{s}$ for all images $s$.

\section{Template-Based Reconstruction for a Single Image}

We start by describing our method for reconstruction from a single image when a template shape is known. This will be later extended to the case where no template is available. We assume that point correspondences between the input image and the template are given.

The geodesic distances of all pairs of points $(i, j) \in \mathcal{N}$ on the $3 \mathrm{D}$ shape to be reconstructed, denoted by $t_{i j}$, are known and obtained from the template shape, as in [2,3], but could alternatively be obtained, for instance, from similar training shapes. As previously mentioned, we assume that: (i) points $(i, j)$ are only neighbours if their distance is small; and (ii) the Euclidean distance is a reasonable approximation of the geodesic distance in a small neighbourhood.

Recall that our method incorporates a soft inextensibility constraint. In order to impose such a constraint, we formulate an energy function that penalises 
deviations from inextensibility. For the case where distances $\left(t_{i j}\right)$ between pairs of points are known, the energy function is of the form:

$$
E(\boldsymbol{\mu})=\sum_{(i, j) \in \mathcal{N}}||\left|Q_{i}\left(\mu_{i}\right)-Q_{j}\left(\mu_{j}\right) \|-t_{i j}\right|
$$

where $\boldsymbol{\mu}=\left\{\mu_{i} \in \mathbb{R}: i=1, \ldots, N\right\}$ and $\left\|Q_{i}-Q_{j}\right\|$ is the euclidean distance between points $i$ and $j$ in the reconstruction.

All the terms in this energy are non-negative and the energy is minimised when the inextensibility constraints are exactly satisfied, i.e. when the euclidean distance between two reconstructed points is the same as the distance between those two points in the template.

\section{Template-Free Reconstruction from Multiple Images}

The energy (1) is only applicable when the distances $t_{i j}$ are known. A more realistic assumption is that a template might not be available but multiple images of the object can be acquired. In this section we assume that the distances between neighbouring points are unknown and estimated together with the depths. Similarly to section 2, our main assumption is that the surface is close to inextensible, so that the following relation should be satisfied for two given images $s$ and $r$ :

$$
\left\|Q_{i}^{s}-Q_{j}^{s}\right\|=\left\|Q_{i}^{r}-Q_{j}^{r}\right\| \quad \forall(i, j) \in \mathcal{N}
$$

Since we assume that more than two images may be available, we introduce auxiliary variables $d_{i j}$ that represent the distance between points $i$ and $j$. We formulate the task of $3 \mathrm{D}$ reconstruction as an energy minimisation problem, where the energy is a function of the form:

$$
E(\boldsymbol{\Lambda}, \boldsymbol{d})=\sum_{s=1}^{S} \sum_{(i, j) \in \mathcal{N}}\left|\left\|Q_{i}^{s}\left(\mu_{i}^{s}\right)-Q_{j}^{s}\left(\mu_{j}^{s}\right)\right\|-d_{i j}\right|
$$

where the quantities to be estimated are $\boldsymbol{\Lambda}=\left\{\mu_{i}^{s} \in \mathbb{R}: s=1, \ldots, S ; i=1, \ldots, N\right\}$, the vector encoding the depths of all points in all images; and $\boldsymbol{d}=\left\{d_{i j} \in \mathbb{R}_{\geq 0}\right.$ : $(i, j) \in \mathcal{N}\}$, the vector of distances between all pairs of neighbouring points. The vector of estimated distances $\boldsymbol{d}$ can be loosely interpreted as a weak template for the underlying (close to inextensible) shape, such that deviations from this template are penalised in the reconstruction of each image.

Reconstruction Ambiguities For a perspective camera, the energy function (3) has a trivial minimum for $\boldsymbol{\Lambda}=0$ and $\boldsymbol{d}=0$. This is due to the $\mathrm{depth} / \mathrm{scale}$ ambiguity inherent to the perspective camera model. In order to avoid this trivial solution, when using a perspective camera, we impose the constraint $\mu_{i}^{s} \geq k \forall s, i$. This constraint can be seen as fixing an arbitrary scale to the object. Note that this trivial minimum only occurs for energy (3) and not for energy (1), since for this case the scale of the object is fixed.

In contrast, the orthographic camera model presents as ambiguities a global depth and flip ambiguity. The scale of the object is invariant under these ambiguities, thus this model does not suffer from the trivial minimum. 


\section{Discrete Optimisation}

We rely on discrete optimisation methods and in particular fusion moves to minimise both the template-based (1) and the template-free (3) energy functions. While (1) is a pairwise energy function, the template-free problem involves a higher-order energy function (3), with cliques of size 3 . We describe in detail how energy (3) is minimised and omit details on the minimisation of (1) since it is analogous, but simpler.

\subsection{Fusion Moves}

Fusion moves $[14,15]$ have been recently introduced as a method for minimising energy functions deriving from Markov Random Fields models. They are particularly suitable for minimising energy functions over continuous variables and they have been successfully applied to stereo [14] and optical flow [15] estimation.

Assume that the goal is to minimize an energy function $E(\boldsymbol{x})$ and that we are given two suboptimal proposals (or solutions) $\boldsymbol{x}^{0}$ and $\boldsymbol{x}^{1}$. A fusion move "fuses" these two solutions into a new one $\boldsymbol{x}^{\text {new }}$ with the following guarantee: $E\left(\boldsymbol{x}^{n e w}\right) \leq E\left(\boldsymbol{x}^{0}\right), E\left(\boldsymbol{x}^{n e w}\right) \leq E\left(\boldsymbol{x}^{1}\right)$, i.e. it produces a solution which has smaller energy than any of the two proposals. The new solution, $\boldsymbol{x}^{\text {new }}$, is obtained by minimising a specially designed binary function $E^{\prime}(\boldsymbol{y})$. For more details on how this binary function is constructed, see [15]. Given the optimal solution, $\overline{\boldsymbol{y}}$, to this binary problem, i.e. $\overline{\boldsymbol{y}}=\arg \min \boldsymbol{y} E^{\prime}(\boldsymbol{y})$, the new solution $\boldsymbol{x}^{\text {new }}$ is constructed as follows: $x_{i}^{\text {new }}=x_{i}^{0}$ if $\bar{y}_{i}=0$ and $x_{i}^{\text {new }}=x_{i}^{1}$ if $\bar{y}_{i}=1$, where $i$ indexes the vector $\boldsymbol{x}$ of unknowns. The form of the binary energy, $E^{\prime}(\boldsymbol{y})$, depends on the original problem. When minimising energy (3) the resulting binary problem is also a sum of triple cliques. We use the reduction introduced in [16] to convert the energy into a pairwise function and minimise it using the QPBO algorithm (see e.g. [17] for a review). This algorithm is not guaranteed to produce an optimal solution if the function is non-submodular. However, even if the global minimum of the binary problem is not achieved, the fusion move is still guaranteed not to increase the energy. We use the QPBO implementation of [17] and the maxflow algorithm of [18] in our experiments.

Similarly to previous approaches, we use fusion moves in an iterative fashion by fusing several proposals.

\subsection{Proposal Generation}

A crucial step for the success of fusion moves is the quality of the proposals. In our experiments we observed that using a chain of proposals, i.e. proposals which are derived sequentially from the previous solution, produces better results than the common strategy of generating independent proposals. We use two different strategies for generating proposals based on a current solution: greedy region growing and gradient descent.

Greedy Region Growing Given a current solution $\left(\boldsymbol{\Lambda}^{\prime}, \boldsymbol{d}^{\prime}\right)$ and a seed region $A \subset\{1, \ldots, N\}$, i.e. $A$ is a subset of feature points, we obtain a new proposal $(\bar{\Lambda}, \bar{d})$ by fixing the depths of the points in the seed region to the current solution. Then, we iterate over all other points, sorted according to their distance to the 


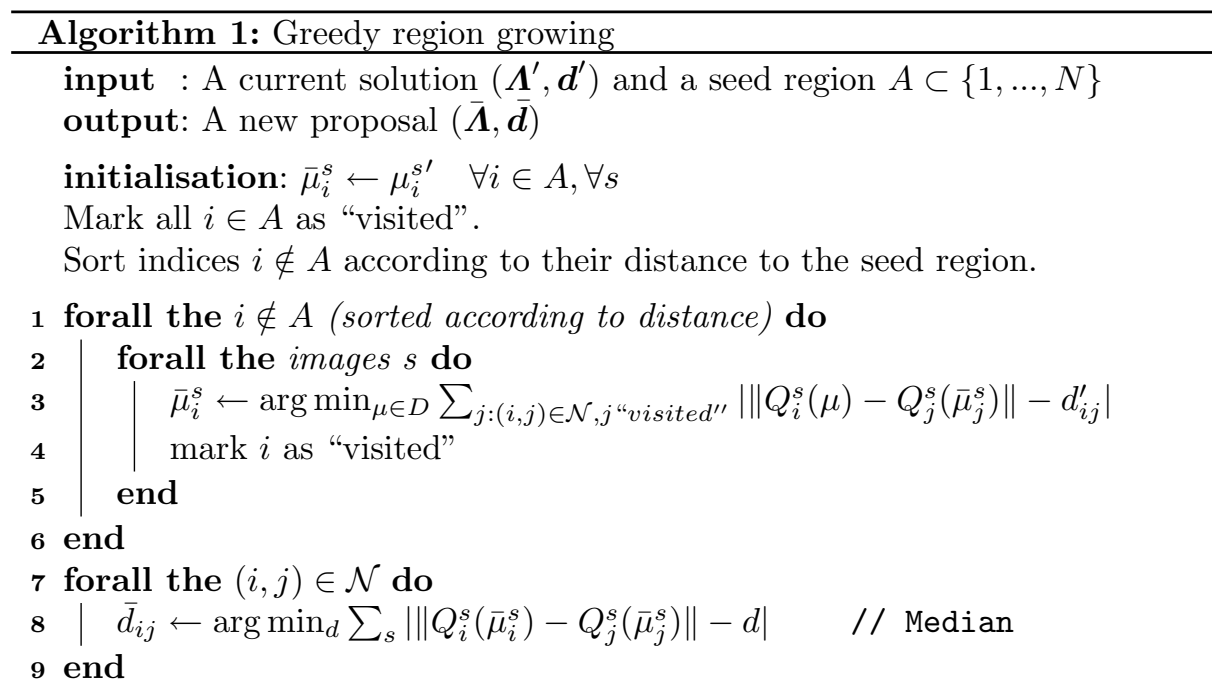

seed region, and update their depth. The new value for the depth is chosen so that the distances between this point and its already visited neighbours better match the current distances $\boldsymbol{d}^{\prime}$. The outline of the algorithm is shown in Algorithm 1. Note that, in the minimisation defined in line 3 of Algorithm 1 we allow only a discrete set $D$ of possible depths in order to improve efficiency. As mentioned in [15], a proposal does not need to be correct in all the points in order to be "useful". Instead, it can still be useful if only a part of it corresponds to a good solution. We expect a proposal generated using this algorithm to satisfy this criterion.

Gradient Descent Another alternative to generate a proposal from a current solution is to adopt a gradient descent strategy as proposed in [19]. The new proposal $(\overline{\boldsymbol{\Lambda}}, \overline{\boldsymbol{d}})$ is obtained from the current one $\left(\boldsymbol{\Lambda}^{\prime}, \boldsymbol{d}^{\prime}\right)$ by differentiating the energy function:

$$
\bar{\mu}_{i}^{s}=\mu_{i}^{s^{\prime}}-\lambda \frac{\partial E(\boldsymbol{\Lambda}, \boldsymbol{d})}{\partial \mu_{i}^{s}} \quad \bar{d}_{i j}=d_{i j}^{\prime}-\lambda \frac{\partial E(\boldsymbol{\Lambda}, \boldsymbol{d})}{\partial d_{i j}}
$$

where $\lambda$ is a weighting term. Note that this weighting is less crucial than in normal gradient descent, since using this new proposal in a fusion move guarantees that the energy does not increase.

\subsection{Initialisation}

We have described how to obtain new proposals from a current solution. We now discuss how to define the initial proposal $\left(\boldsymbol{\Lambda}^{\prime}, \boldsymbol{d}^{\prime}\right)$. We start by approximating the unknown distances $d_{i j}^{\prime}$ from the known 2D projections by taking the maximum distance in $2 \mathrm{D}$ between each pair of points in the image, i.e. we initialise the 
distances as $d_{i j}^{\prime}=\max _{s}\left|p_{i}^{s}-p_{j}^{s}\right|$. For an orthographic camera, these 2D distances are guaranteed to be a lower bound on the real $3 \mathrm{D}$ distances.

All the depths are initialised to the same value, i.e. we set $\mu_{i}^{s \prime}=$ const, $\forall i, s$, where const $=0$ for orthographic and const $=k$ for perspective cameras.

\subsection{Full Optimisation Method}

Our overall algorithm functions in the following way: we initialise the algorithm 8 times as described in section 4.3. We then run 50 fusion moves for each initialisation, where the proposals are generated using the greedy region growing procedure summarised in Algorithm 1. As choice of seed region we alternate between using a pair of neighbouring points or a half-plane region defined by a constraint of the form: $\left\{i: x_{i}^{s}<m\right\},\left\{i: x_{i}^{s}>m\right\},\left\{i: y_{i}^{s}<m\right\}$ or $\left\{i: y_{i}^{s}>m\right\}$ for some frame $s$ and constant $m$.

The 8 initialisations are optimised independently, therefore we obtain 8 different solutions and we choose the one with minimum energy. Finally, we run an additional 100 fusion moves on this solution with proposals obtained from gradient descent.

\section{Extensions}

\subsection{Additional Priors}

Two commonly used priors for the task of non-rigid reconstruction are temporal and surface smoothness. Both these priors can be included in our formulation by adding extra-terms to the energy function and using a similar optimization strategy. Note that, the addition of these extra-terms requires a choice of sensible weights for the different terms.

Temporal Smoothness Temporal smoothness is a common assumption in reconstruction from video sequences [5]. Note that so far we have not assumed any particular order in the images used. If these are consecutive frames of a video sequence, our formulation can be complemented with a temporal prior by adding one of the following terms to the energy function:

$$
T(\boldsymbol{\Lambda})=\sum_{s=1}^{S-1} \sum_{i=1}^{N}\left|\mu_{i}^{s}-\mu_{i}^{s+1}\right| \quad T(\boldsymbol{\Lambda})=\sum_{s=1}^{S-2} \sum_{i=1}^{N}\left|\mu_{i}^{s}-2 \mu_{i}^{s+1}+\mu_{i}^{s+2}\right|
$$

that correspond respectively to a first-order and a second-order temporal prior.

Surface Smoothness Surface smoothness is usually imposed in approaches that recover a continuous surface [3] or that represent the reconstruction as a combination of smooth basis shapes [1]. Since we do not recover a continuous surface or use a basis shape formulation, we use an alternative definition for smoothness that follows [14] and that has been shown to considerably improve the quality of stereo reconstructions. Assume the feature points form a regular square grid. We consider triplets of neighbouring points $(i, j, k)$ on the same horizontal or vertical line. A second-order smoothness prior can be imposed by adding extra terms, one per triplet, to the energy function. The term for each 
triplet has the form: $\left|\mu_{i}-2 \mu_{j}+\mu_{k}\right|$, where $j$ is the index of the middle point and we assume for simplicity that the camera is orthographic.

For most sequences no prior knowledge exists on the location of feature points and they do not necessarily form a regular grid. In that case we approximate this construction using triplets of nearby points which are collinear in all images.

\subsection{Dealing with Missing Data}

Our formulation does not require that all points are visible in all frames and can be easily updated to deal with missing data. Let $V_{s}$ be an indicator function for each image $s$, such that $V_{s}(i)=1$ if point $i$ is visible in image $s$ and $V_{s}(i)=0$ otherwise. Also, let $\mathcal{N}_{s}=\left\{(i, j):(i, j) \in \mathcal{N} \wedge\left(V_{s}(i)=1\right) \wedge\left(V_{s}(j)=1\right)\right\}$ be a constrained neighbourhood system for each image $s$ that takes visibility into account. The energy function in the presence of missing data takes the form:

$$
E(\boldsymbol{\Lambda}, \boldsymbol{d})=\sum_{s=1}^{S} \sum_{(i, j) \in \mathcal{N}_{s}}||\left|Q_{i}^{s}\left(\mu_{i}^{s}\right)-Q_{j}^{s}\left(\mu_{j}^{s}\right) \|-d_{i j}\right|
$$

where $\mu_{i}^{s}$ is not defined if $V_{s}(i)=0$, i.e. there is no $3 \mathrm{D}$ reconstruction if the point is occluded. Adapting the optimisation method to deal with missing data is also straightforward, since it only requires taking into account the per-image neighbourhood system in the region growing algorithm.

\section{Experiments}

We evaluate our approach in the two different scenarios it allows: template based reconstruction for a single image and template-free reconstruction from multiple images. We use several datasets that have been previously introduced in $[2,8,20$, 11]. We start by providing details about the construction of the neighbourhood system and the computation of the error measures.

Definition of the Neighbourhood System $\mathcal{N}$ For all pairs of points $(i, j), 1 \leq$ $i \neq j \leq N$ we compute their furthest distance $f_{i j}$ over all the frames: $f_{i j}=$ $\max _{s}\left\|p_{i}^{s}-p_{j}^{s}\right\|$. For each point $i$, let $K_{i}$ be its set of $k$-nearest neighbours according to the distances $f_{i j}$. The neighbourhood system is then defined as $\mathcal{N}=\cup_{i}\left\{(i, j): j \in K_{i}, f_{i j} \leq t\right\}$, where $t$ corresponds to a threshold on the distance. For most of the experiments we set $k=10$ and $t=200$. An example of neighbourhood system can be seen in Fig.1(b), where each edge corresponds to a pair of points in the neighbourhood.

Error Measures Some of the datasets have associated ground truth that can be used to quantitatively evaluate our method. We denote the ground truth reconstruction of point $i$ in image $s$ by $\hat{Q}_{i}^{s}$. We found that there is no standard error measure uniformly used in previous related work and in order to enable an easier comparison we use two different error metrics reported by previous authors: per-frame pointwise reconstruction error (PWRE) [2,3], given by $\mathrm{PWRE}=\frac{1}{N} \sum_{i=1}^{N}\left\|\hat{Q}_{i}^{s}-Q_{i}^{s}\right\|$; and root mean squared error (RMSE) over a sequence [8] defined as RMSE $=\sqrt{\frac{1}{3 N S} \sum_{s=1}^{S} \sum_{i=1}^{N}\left\|\hat{Q}_{i}^{s}-Q_{i}^{s}\right\|^{2}}$. When considering an orthographic camera, we resolve the depth ambiguity by subtracting the centroids 
PWRE for our method

\begin{tabular}{l|cc} 
& No noise & With noise \\
\hline Orthographic & 0.25 & 0.77 \\
Perspective & 0.39 & 1.80
\end{tabular}
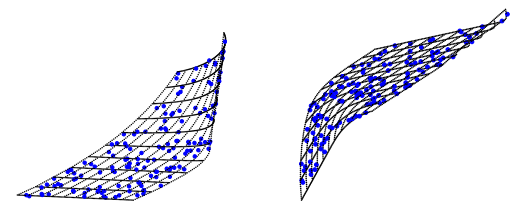

Fig. 3. Template based reconstruction for synthetic data. The table records median PWRE for 1000 randomly generated paper sheets. We show results for two typical paper sheets: black grid is ground truth and blue points are our reconstruction.

from both the ground truth and our solution. We then choose the flip with smallest error, from the two possible flips. In contrast with some previous work we do not use Procrustes analysis to align the shapes.

\subsection{Template Based Reconstruction for a Single Image}

To evaluate the performance of our algorithm for 3D reconstruction of a single image given a known template, we use the synthetic dataset provided by $[2,3]$, based on synthetically generated pieces of paper (i.e. developable surfaces). We use the method in [21] and the author's code, to generate 1000 synthetic pieces of paper. Furthermore, we use the same experimental set up as reported in [3]: a $200 \mathrm{~mm}$ wide piece of paper with 150 randomly extracted correspondences and added zero mean Gaussian noise with standard deviation of 1 pixel to the point correspondences.

The results for our method are summarised in Fig. 3. We consider both an orthographic and a calibrated perspective camera and generate the images accordingly. We set $k=20$ in the generation of the neighbourhood. Our 3D error for perspective projection with noise compares favourably with the results reported in [3]. We outperform all other point-wise reconstruction methods [9, 2 , $3]$, all of which have a median PWRE greater than 2 while ours is 1.8. The only method that outperforms ours is the surface reconstruction method also introduced in [3] which has median PWRE around 1.5 (see figure 3 in [3]). However, surface reconstruction methods use stronger priors than pointwise approaches.

\subsection{Template-Free Reconstruction of a Small Collection of Images}

We show results of our template-free reconstruction algorithm applied to a small number of images. We report qualitative results on three datasets with real images often used in the NRsfM literature.

Graffiti dataset [3]: Fig. 1 shows the reconstructions we obtained by using only the two images displayed.

Paper sequence [20]: Fig. 4 shows the results of our method applied to reconstruct just 3 frames of the sequence. We use the tracks provided by [8].

In these reconstructions we make use of a perspective calibrated camera and of the surface smoothness prior discussed in section 5.1, since the interest points are positioned in a grid. For both datasets, we obtain reconstructions which are visually comparable to previous methods that use more information, either a 


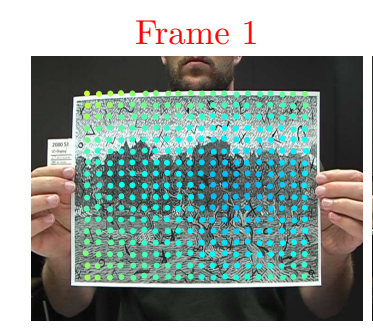

Frame 1

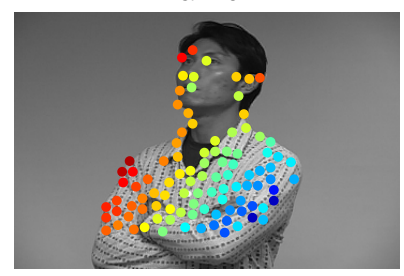

Frame 30

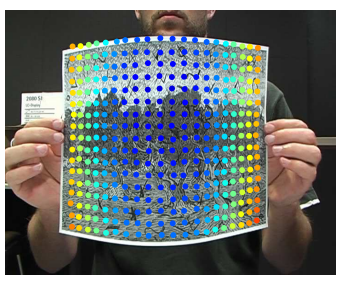

Frame 39

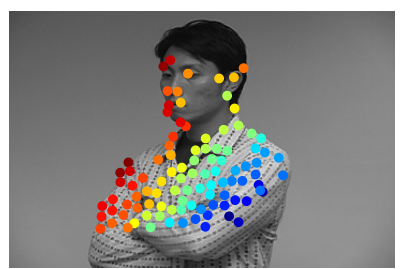

Frame 70

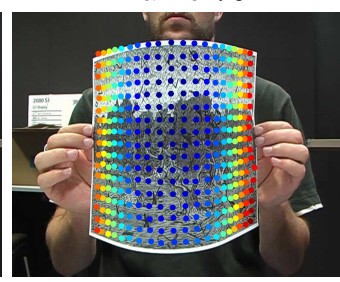

Frame 60

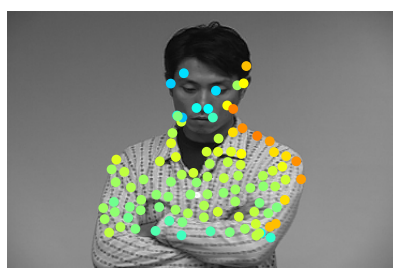

Fig. 4. Reconstruction of a small collection of images. Original images with point correspondences colour coded by estimated depth - red points are further from the camera and blue points are closer. Top row: Paper sequence with estimated 3D shapes for the 3 frames. Bottom row: Head sequence. Best viewed in colour

template [3] or temporal consistency $[8,6]$. Furthermore, our method recovers correctly the relative depths of the reconstructed shapes. This can be seen in the last column of Fig. 4.

Head sequence [22]: This is an example of a non-developable surface with articulated motion. We reconstruct the three frames shown in Fig. 4. The choice of the neighbourhood system is particularly relevant for this sequence, since a naive choice can connect points which are in different articulated parts (for example the shoulder and the ear). In order to prevent this, we set the parameters to be $t=100$ and $k=7$. We correctly recover the differences in depth between the two upper-arms in the first two frames and the facial features.

\subsection{Reconstruction of Temporal Video Sequences}

We also evaluate our method in four challenging MoCap temporal sequences (with ground truth data). The wind [8] and flag [11] sequences correspond respectively to paper and fabric waving in the wind, the jacky [5] sequence shows a face and the bend [8] sequence depicts a paper bending. We assume an orthographic camera and report results without the use of the temporal prior described in section 5.1. The results are summarised in Table 2. Following [8], we also report results when Gaussian noise of standard deviation $\sigma=\frac{\rho}{100} \beta$ is added to every point, where $\beta$ is the maximum distance of an image point to the centroid of all the points. Our method consistently outperforms Taylor et al. [8] on all ground truth sequences, and performs comparably to Russell et al. $[6]^{1}$. On the jacky sequence we also get comparable results with methods that use linear

${ }^{1}[8,6]$ are currently considered the state of the art NRsfM methods. 


\begin{tabular}{c|c|c|c|c|c|c}
\hline Sequence & $\mathbf{S}$ & $\mathbf{N}$ & $\begin{array}{c}\text { Noise } \\
\boldsymbol{\rho}\end{array}$ & $\begin{array}{c}\text { Time } \\
(\mathbf{m i n})\end{array}$ & Ours & $\begin{array}{c}\text { RMSE } \\
\text { Competitors }\end{array}$ \\
\hline flag [11] & 50 & 540 & 0 & 12 & 1.79 & $1.29[6]^{*} / 2.13[8]^{*}$ \\
\hline \multirow{2}{*}{ wind [8] } & \multirow{2}{*}{1000} & \multirow{2}{*}{17} & 0 & 15 & 0.73 & $1.7[8]^{\dagger}$ \\
& & & 1 & 10 & 1.48 & $3.4[8]^{\dagger}$ \\
\hline \multirow{2}{*}{ jacky [5] } & \multirow{2}{*}{1000} & \multirow{2}{*}{43} & 0 & 40 & 3.14 & $3.8[8]^{\dagger} / 3.2[5]^{\dagger}$ \\
& & & 1 & 49 & 3.85 & $4.7[8]^{\dagger} / 3.5[5]^{\dagger}$ \\
\hline bend [8] & 471 & 34 & 0 & 11 & 0.87 & - \\
\hline
\end{tabular}

*Error value reproduced from [6]. This error corresponds to the full sequence and it was converted from the measure used in [6]: the Frobenius norm normalised by size. ${ }^{\dagger}$ Error value reproduced from [8].

Table 2. Quantitative results for MoCap temporal sequences. We compare our method with previous methods in terms of RMSE. For the flag sequence, we reconstructed only the first 50 frames.
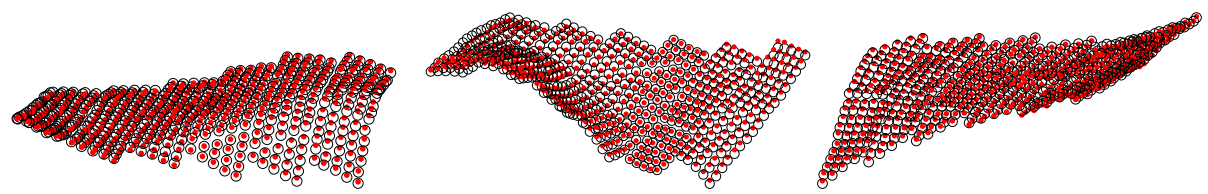

Fig. 5. Flag sequence. Reconstruction (red dots) overlaid with ground truth (black circles) for a few frames from different viewpoints.

basis shapes [5]. The near rigidity of this sequence is particularly favourable for this type of methods. On the challenging MoCap flag sequence our 3D error of 1.79 , on the first 50 frames of the sequence, is only slightly larger than the one given by the piecewise quadratic approach of [6] with a reported error of 1.29 for the full sequence. We show qualitative 3D reconstructions for the flag sequence in Fig. $5^{2}$.

\section{Conclusion}

In this work, we introduced a soft inextensibility constraint for reconstruction of non-rigid objects from as few as two images. We showed how we can include inextensibility in an energy minimisation framework and how to optimise it using recently introduced techniques for discrete optimisation. Our method can be used in two distinct scenarios that have so far been considered separately with specially designed methods: template based reconstruction and NRSfM. We evaluated our method in several datasets, including MoCap sequences, obtaining similar performance to state-of-the-art methods. Our formulation allows the incorporation of additional priors and we believe it can be further extended and customised.

${ }^{2}$ Note that the apparent strip-like structure of the flag is present in the ground truth

$3 \mathrm{D}$ and is due to the regular way in which the MoCap markers were placed. 


\section{References}

1. Salzmann, M., Moreno-Noguer, F., Lepetit, V., Fua, P.: Closed-form solution to non-rigid 3d surface registration. In: ECCV. (2008)

2. Perriollat, M., Hartley, R., Bartoli, A.: Monocular template-based reconstruction of inextensible surfaces. In: BMVC. (2008)

3. Brunet, F., Hartley, R., Bartoli, A., Navab, N., Malgouyres, R.: Monocular template-based reconstruction of smooth and inextensible surfaces. In: ACCV. (2010)

4. Bregler, C., Hertzmann, A., Biermann, H.: Recovering non-rigid 3D shape from image streams. In: CVPR. (June 2000)

5. Torresani, L., Hertzmann, A., Bregler., C.: Non-rigid structure-from-motion: Estimating shape and motion with hierarchical priors. PAMI (2008)

6. Russell, C., Fayad, J., Agapito, L.: Energy based multiple model fitting for nonrigid structure from motion. In: CVPR. (2011)

7. Varol, A., Salzmann, M., Tola, E., Fua, P.: Template-free monocular reconstruction of deformable surfaces. In: ICCV. (2009)

8. Taylor, J., Jepson, A.D., Kutulakos, K.N.: Non-rigid structure from locally-rigid motion. In: CVPR. (2010)

9. Salzmann, M., Fua, P.: Reconstructing sharply folding surfaces: A convex formulation. In: CVPR. (2009)

10. Ferreira, R., Xavier, J., Costeira, J.P.: Shape from motion of nonrigid objects: the case of isometrically deformable flat surfaces. In: BMVC. (2009)

11. Fayad, J., Agapito, L., Del Bue, A.: Piecewise quadratic reconstruction of non-rigid surfaces from monocular sequences. In: ECCV. (2010)

12. Ullman, S.: Maximizing rigidity: the incremental recovery of 3-d structure from rigid and rubbery motion. Perception (1984)

13. Jasinschi, R., Yuille, A.: Nonrigid motion and regge calculus. Journal of the Optical Society of America 6(7) (1989) 1088-1095

14. Woodford, O.J., Torr, P.H.S., Reid, I.D., Fitzgibbon, A.W.: Global stereo reconstruction under second order smoothness priors. In: CVPR. (2008)

15. Lempitsky, V., Rother, C., Roth, S., Blake, A.: Fusion moves for markov random field optimization. PAMI (8) (2010) 1392-1405

16. Ishikawa, H.: Higher-order clique reduction in binary graph cut. In: CVPR. (2009)

17. Rother, C., Kolmogorov, V., Lempitsky, V., Szummer, M.: Optimizing binary MRFs via extended roof duality. In: CVPR. (2007)

18. Boykov, Y., Kolmogorov, V.: An experimental comparison of min-cut/max-flow algorithms for energy minimization in vision. PAMI (September 2004)

19. Ishikawa, H.: Higher-order gradient descent by fusion-move graph cut. In: ICCV. (2009)

20. Salzmann, M., Hartley, R., Fua, P.: Convex optimization for deformable surface 3-d tracking. In: ICCV. (2007)

21. Perriollat, M., Bartoli, A.: A quasi-minimal model for paper-like surfaces. In: CVPR. (2007)

22. Yan, J., Pollefeys, M.: A general framework for motion segmentation: Independent, articulated, rigid, non-rigid, degenerate and non-degenerate. In: ECCV. (2006) 